\title{
Hug Over a Distance
}

\author{
Florian 'Floyd'
Mueller', \\ Frank \\ Martin R. \\ Vetere $^{1}$ \\ Gibbs $^{1}$ \\ $\underset{\text { Kjeldskov }^{1,3}}{\text { Jesper }} \quad$ Sonja Pedell $^{1} \quad$ Steve Howard $^{1,3}$ \\ ${ }^{1}$ Interaction Design Group, \\ ${ }^{2}$ CSIRO \\ ${ }^{3}$ Aalborg University \\ Department Of Information Systems \\ University of Melbourne \\ Victoria 3010, Australia \\ Commonwealth Scientific and \\ Industrial Research Organisation \\ Australia \\ Fredrik Bajers Vej 5 \\ 9100 Aalborg \\ Denmark \\ floyd@exertioninterfaces.com, fv@unimelb.edu.au, martinrg@unimelb.edu.au, jesper@cs.auc.dk, \\ s.pedell@pgrad.unimelb.edu.au, showard@unimelb.edu.au
}

\begin{abstract}
People in close relationships, who are separated by distance, often have difficulty expressing intimacy adequately. Based on the results of an ethnographic study with couples, a prototype was developed to test the feasibility of technology in the domain of intimacy. Hug Over a Distance is an air-inflatable vest that can be remotely triggered to create a sensation resembling a hug. Although the couples did not consider the vest to be useful in their daily lives, the prototype served to provoke and stimulate design ideas from the couples during participative design workshops. An additional and unexpected benefit was also found: the prototype enhanced the couples' understanding of the researchers' methods, suggesting that prototypes can serve as tools to make participatory design volunteers aware of their importance in academic research.
\end{abstract}

\section{Author Keywords}

Social interaction, haptic interface, tactile display, remote interaction, intimacy, participatory design, wearable computing, ubiquitous computing, design workshop.

\section{ACM Classification Keywords}

H5.2. Information interfaces and presentation (e.g., HCI): User Interfaces.

\section{INTRODUCTION}

Task-oriented technology, such as email, videoconferencing and mobile phone voice and text communications were initially invented to increase efficiency in communication and collaboration. However, people often adapt them to satisfy their needs for social interaction: Flirting, chatting or finding marriage partners with these technologies are all lucrative businesses today, even though the initial designers surely did not envision such uses.

Copyright is held by the author/owner(s).

CHI 2005, April 2-7, 2005, Portland, Oregon, USA.

ACM 1-59593-002-7/05/0004.

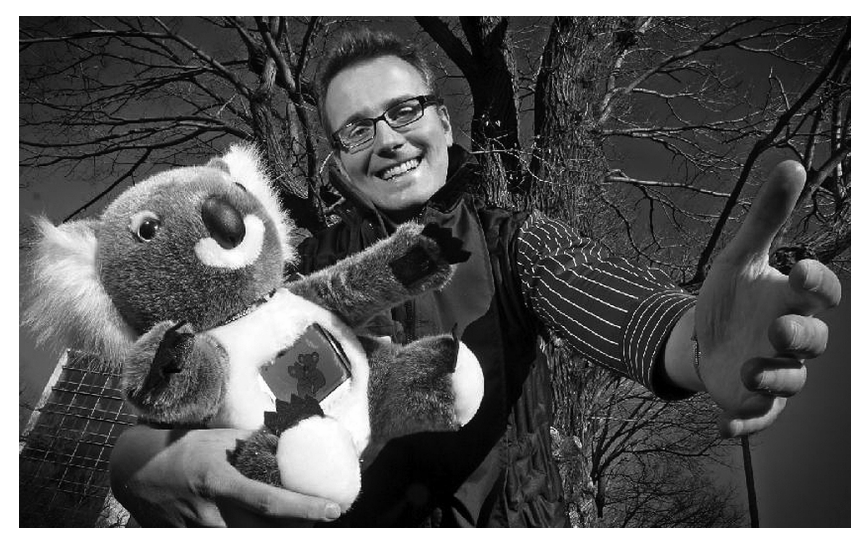

Figure 1. Rubbing the koala sends a wireless Hug over a Distance executed through the vest.

\section{RESEARCH QUESTION}

Through the use of focus groups and design workshops, we sought to answer the question: What would an interface look like that is designed from the outset to facilitate and encourage social, even intimate interaction?

\section{Research Plan}

In our initial empirical study using focus groups and cultural probes, we examined personal interactions of six intimate couples [6]. We then set out to design and build a prototype that addresses some of the participants' needs. We identified two of the major themes, designed an interface around these themes, and decided to present this prototype to the participants during a design workshop. We were hoping to receive feedback and provoke design ideas from the participants by the use of the prototype.

Two of the major themes identified in the study were:

a) "Haptic": Participants expressed the desire for a tactile or haptic experience when communicating with their partner remotely. They expressed the need for an intimate "feel good" palpable experience, very different to the "alert" functionality of vibrating devices, such as mobile phones. 
b) "Unobtrusive": Couples liked sending small "I love you" text messages, (which we call "emotional pings"), because they were short and private, can be sent and received everywhere, and do not necessitate an immediate response. However, these messages were often received at inopportune moments or read too late. The couples also used secret codes to not reveal intimate messages to their surroundings, such as 143 for the amount of letters in the words "I love you". Some of the participants expressed a desire to be able to send a "kiss across the Internet" [4], which would be received immediately, yet did not unduly interrupt their partner.

\section{REQUIREMENTS}

We selected these two themes, "haptic" and "unobtrusive", for our requirements because we believe they hold the most potential for a novel and thought-provoking interface. Therefore, our new interface a) allows for a tactile interaction with an emotional content, similar to holding hands or giving a hug, and b) can be used anywhere, anytime, without interrupting the receiver.

\section{Use Scenario}

An intimate couple is separated from each other due to a work assignment. He is working in a city far away from home, and he misses his partner. He misses giving her a hug and holding hands. Therefore, he picks up his mobile phone to call her, but hesitates, because she is at work and a call might disturb her. Besides, he does not really have anything important to say and decides not to contact her. She, however, misses him, and wishes he would get in touch. Hence, the communication diminishes.

Then he decides to send her a Hug Over a Distance: He takes the koala (see Figure 2), and rubs its belly. While thinking about his partner, he sends a hug. His partner is wearing a stylish vest, which nobody around her can identify as a piece of wearable computing. However, the vest contains a wireless receiver, which receives the hug request. Inside the vest are small air compartments which inflate quickly all around her body torso, giving a sensation similar to a real hug. Although she is at work, the hug is discretely received, because others cannot "see" the hug.

She knows that he was thinking about her because she received a tactile emotional message from him, meaning "I am thinking about you." They feel closer although they are still apart.

\section{Motivation}

It is important for us to emphasize that our intention is not to recreate accurately the physical and emotional experience of a real hug. Rather, we want to demonstrate, using a piece of wearable computing, that it is possible to send an "emotional ping" to a remote loved one with a tactile and unobtrusive interface. We are referring to a "hug", because the feeling of light pressure around the body, combined with the associated warmth it creates, most closely resembles a hug.

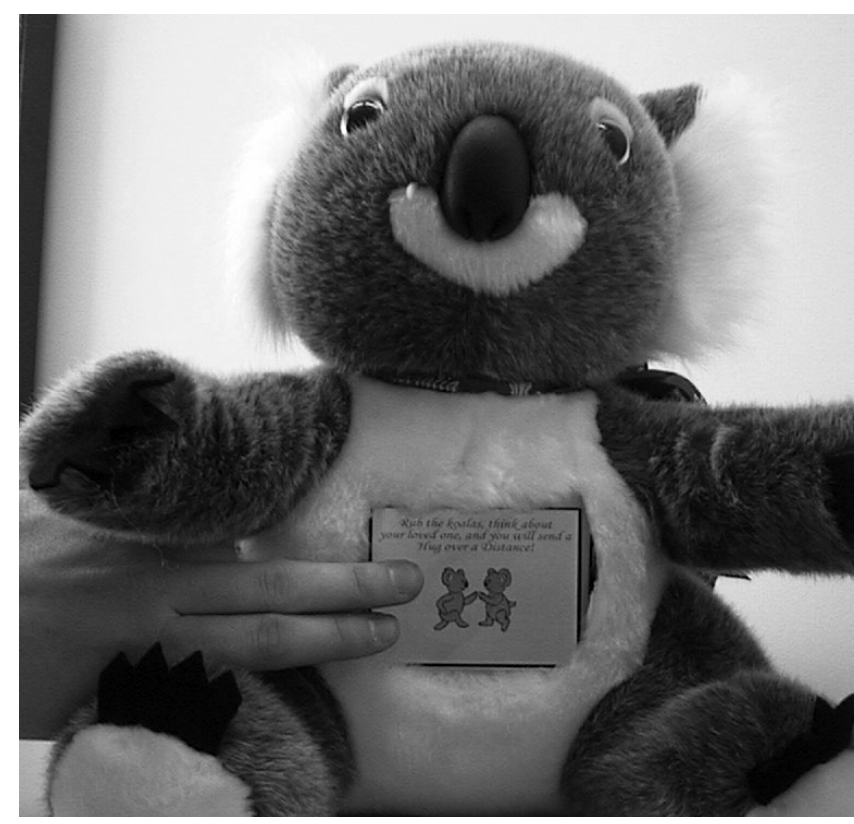

Figure 2. The wireless koala is currently a placeholder for a second vest.

\section{IMPLEMENTATION}

The koala contains a Personal Digital Assistant (PDA), which displays an animation of two koalas that will hug each other if a user rubs the PDA's touch sensitive screen. The PDA communicates wirelessly with a second PDA inside the vest (Figure 3) using TCP/IP via 802.11 or via Bluetooth through the users' phones. When the vest receives a hug request, it communicates through its serial port to a serial I/O controller, which opens a relay that starts an air compressor. The inside of the vest is lined with air compartments that cover the entire upper body torso. When they are filled with air from the compressor, the wearer experiences a light, but palpable pressure around her/his body. Four seconds later, an electronic valve opens and releases the air. The PDA sends an acknowledgement back to the koala, indicating the hug was received, and thanks the sender with a kissing sound.

\section{Limitations}

The aim of the design is to facilitate an intimate experience, whilst not distracting other people, unlike the ring tone of a mobile phone. In order to create a feeling similar to a hug, we use a compressor to inflate the vest rapidly and with high pressure. The compressor is quite powerful, but in the initial prototype generates a loud noise during inflation.

The koala is a preliminary interface to initiate a hug. Ideally, we would like to develop two vests instead of using the koala and thus enable two-way interaction: a hug can be initiated by miming a hug gesture, and, using a second vest, reciprocated. 


\section{DESIGN WORKSHOP}

Aims

A design workshop was held to generate design ideas on how technology can facilitate intimacy over a distance. Six couples in long-term relationships participated, and two children were also present. We had two reasons for presenting the Hug Over a Distance prototype at the beginning of the design workshop:

- Having gathered the participants' requirements for advantageous technology that facilitates intimacy, we came up with our own design idea of the Hug Over a Distance vest. We wanted to see the reaction of the participants, and find out how closely aligned our idea was to their needs.

- Previous focus groups with the participants elicited rather conventional ideas, mostly variations from gadgets they have seen in movies. Alternatively, their suggestions were either available as a product today or represented iterative steps to improve current technology. As researchers, we were more interested in rather unusual design ideas. We hoped that presenting the participants with a fairly unconventional prototype might spark their creativity.

\section{Reaction to the Prototype}

We demonstrated the Hug Over a Distance vest to the participants, and the initial reaction was amusement. They tried the vest on themselves, some of them several times. The experience of having pressure applied to the upper torso by a vest, instead of a real person during a hug, was described as "weird", and some participants found it difficult to imagine it used in practice. None of the couples wanted one for themselves. On the other hand, they also expressed that exchanging hugs was an interesting idea and that the tactile interface was fun. The couples also expressed that they missed some kind of mutuality (e.g. both partners wearing synchronized vests) because giving someone a hug is a two-way interaction. We expected this reaction, but decided to initially develop a single vest in order to get an early reaction.

The participants did not seem to be able to separate the interface from the noise factor. Although being advised that the loud compressor was only a first implementation tool to get the pressure level right (and can be replaced by more silent technology), the participants repeatedly mentioned the unfeasibility of the generated noise.

One interesting comment was the issue of control on the receiver's end: with current hugs, people can reject a hug. However, with our prototype, people have no choice than accepting the hug (besides taking the vest off), the participants pointed out.

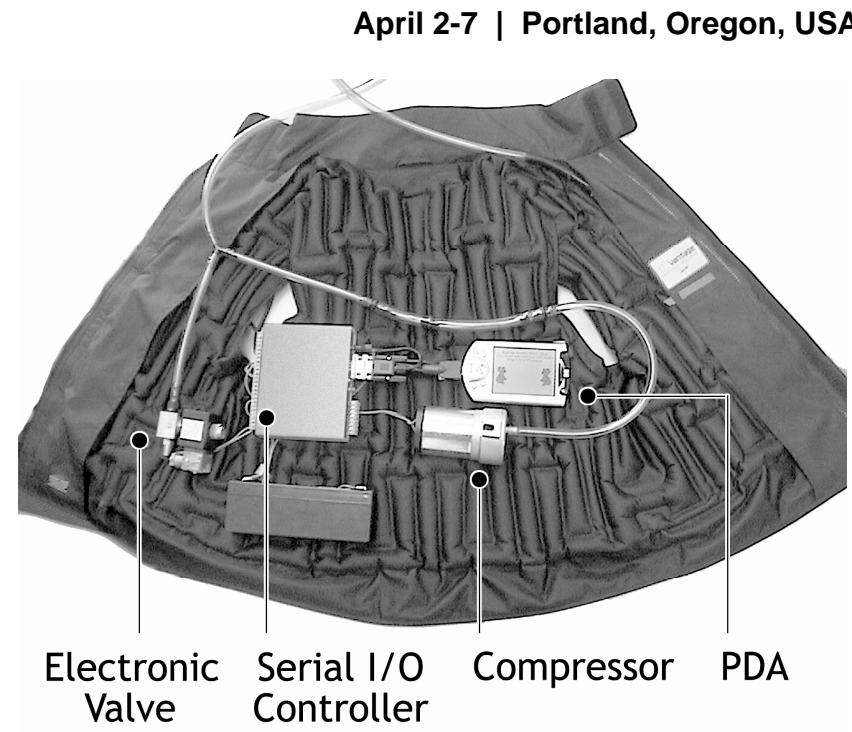

Figure 3. The vest with the technology exposed.

The joyful atmosphere created by the presentation of the prototype, that continued even after the workshop ended, seemed to indicate that the Hug Over a Distance addresses the participants' desire for playfulness and fun in a relationship, as identified in our initial study.

\section{Design Ideas after Experiencing the Prototype}

While the couples were mainly laughing about the vest during the demonstration, they made serious comments and started a far more refined and "spot on" discussion after they had tried it on. We believe the prototype acted as a tactile demonstrator, conveying ideas to the couples in a more "user-friendly", non-technical way.

\section{RESULTS}

We anticipated more creative ideas coming from the participants after having experienced the Hug Over a Distance prototype, but were also aware of the complexity of assessing creativity and comparing creative ideas [5]. However, it was not the quality or quantity of ideas the couples brought forward after having seen the vest (for example, an intelligent sock-drawer that delivers love letters), it was something else that surprised us and made us, not only them, think "outside-the-box":

The couples laughed when they saw the Hug Over a Distance vest, but simultaneously, they also seemed to have had an eye-opening experience towards our methods, processes and research aims. It seemed that from this point, the purpose of all probe activities (the diaries, the scrap books, the photographs, etc) [6] became clearer for the participants. They came to understand that the probe data was collected in order to generate ideas and opportunities. Even though this goal was initially communicated to the participants, they seem to only realize its importance after having seen the prototype.

The participants expressed that the prototype enhanced their understanding of our methods, and they were beginning to 
realize why they were needed in an academic project, why their input was valuable and where this can lead them to. The couples seemed to realize that the previous discussions were not purely academic exercise, but could lead to tangible results that can affect their daily lives in the future. This realization changed also the general atmosphere during the meetings between the participants and the researchers from previously rather stiff to more relaxed and jolly, making it a more enjoyable experience for everybody. We are now planning on introducing a prototype in future workshops not so much as a demonstrator about possible outcome, but simply as an ice-breaker between nonacademic people and academics.

It seemed to have helped the couples understand some of our theoretical concepts better and allowed them to discuss and evaluate them more directly and openly by having seen a physical prototype. One of the participants said: "Now I understand why we did all this."

\section{RELATED WORK}

The importance of hugs for social interactions has been investigated before: "The Hug" [1] acknowledges the human need for physical closeness in remote communications. This product concept describes two connected pillows, fitted with sensors, heat emitters and vibrators, with the aim of connecting people socially. The "HugJackets" [3] are fitted with LEDs that light up if two people wearing them hug each other. The jackets also elicit a "bombastic sound", aiming to actualize the energy transfer during a hug. The "F+R Hugs" project interviewed people in regards to their experience with hugs, and describes a concept to design underwear that is fitted with sensors to emulate a hug [8].

Applying pressure to the upper body torso has also other applications: A pressure vest "simulating a hug" such as in [7] is often used to calm children with autism [2]. Much lighter pressure is utilized by the "White Stone" [9]: it extends the sense of touch over a distance in order to create the feeling of presence with paired "stones". The design concept explains that if one is held in the hand, the corresponding stone should beep and become warm, supposedly eliciting intimacy.

\section{FUTURE WORK}

Based on the feedback with regards to the noise mentioned above, we are now experimenting with small compressed gas cylinders used for quick inflation of racing bikes to overcome the noise factor.

The koala will be replaced with a second vest, which will then allow for a two-way mutual interaction, and remove the abstract layer of the current hug-initiation.

\section{CONCLUSION}

Hug Over a Distance is an interface example for couples to facilitate intimacy when they are apart. Hug Over a Distance supports the delivery of the "soft" feeling of a hug in an unobtrusive way through a remotely controllable airinflatable vest, making it one of very few interfaces that are specifically designed to facilitate intimacy over a distance. This vest was used at a design workshop with couples in intimate relationships where it was used to provoke creative ideas by the participants; however, they did not see the current prototype as a useful item in their daily lives.

The surprising result of this study was that the prototype seemed to have enhanced the couples' understanding of the researchers' methods and purposes, bridging the gap between participants' daily lives and abstract academic concepts. Our results suggest that prototypes can be a valuable tool for stimulating novel design ideas in participatory design activities, and also make volunteers aware of their role and importance in participative design research.

\section{ACKNOWLEDGMENTS}

Figure 1 by Nicole Cleary from mx magazine. Supported by the Smart Internet Technology Cooperative Research Centre and the Danish Technical Research Council.

\section{REFERENCES}

1. Gemperle, F., DiSalvo, C., Forlizzi, J., Yonkers, W. The Hug: a new form for communication. Proc. Designing for User Experiences 2003, ACM Press (2003), 1-4.

2. Grandin, T. Teaching Tips for Children and Adults with Autism. http://www.cdrcp.com/aut_teachingtips.html

3. HugJackets. Studio 5050, New York, USA, http://www.5050ltd.com/hugJackets.html

4. Kaye, J. Making Scents - Aromatic Output for HCI. Interactions 11(1) Jan + Feb 2004, ACM Press (2004), p. $48-61$

5. Kerr, B., Gagliardi, C. Measuring Creativity in Research and Practice.

http://courses.ed.asu.edu/kerr/measuring_creativity.rtf

6. Kjeldskov, J., Vetere, F., Gibbs, M., Howard. S., Pedell, S., Mecoles, K. and Bunyan, M. Mediating Intimacy: designing technologies to support strong tie relations. OZCHI 2004.

7. Paskind, J., Krishnamurty, S. Portable Intelligent Deep Pressure Vest. http://nsf-pad.bme.uconn.edu/2001/ University\%20of\%20Massachusetts\%20Amherst.pdf ,252-253.

8. Rosella, F., Sakai, R. F \& R Hugs. http://projects.interactionivrea.it/2002/desire/f+rhugs/F+R HUGS.pdf

9. Tollmar, K., Junestrand, S., Torgny, O. Virtually living together. Proc. DIS 2000, ACM Press (2000), 83 - 91. 